\title{
Conformal Symmetry and Pion Form Factor: Soft and Hard Contributions
}

\author{
Ho-Meoyng $\mathrm{Choi}^{a}$ and Chueng-Ryong $\mathrm{Ji}^{b}$ \\ ${ }^{a}$ Department of Physics, Teachers College, Kyungpook National University, Daegu, Korea 702-701 \\ ${ }^{b}$ Department of Physics, North Carolina State University, Raleigh, NC 27695-8202
}

\begin{abstract}
We discuss a constraint of conformal symmetry in the analysis of the pion form factor. The usual power-law behavior of the form factor obtained in the perturbative QCD analysis can also be attained by taking negligible quark masses in the nonperturbative quark model analysis, confirming the recent AdS/CFT correspondence. We analyze the transition from soft to hard contributions in the pion form factor considering a momentum-dependent dynamical quark mass from an appreciable constituent quark mass at low momentum region to a negligible current quark mass at high momentum region. We find a correlation between the shape of nonperturbative quark distribution amplitude and the amount of soft and hard contributions to the pion form factor.
\end{abstract}

\section{INTRODUCTION}

The pion elastic form factor has been exemplified by many calculations to understand the substructure of hadrons in terms of the quark-gluon degrees of freedom [1, 2, 3, 4, 5, 6, 6, 8, 9, 10, 11]. Since the valence structure of the pion is relatively simple, the value of the four-momentum transfer square $Q^{2}$ above which a perturbative $\mathrm{QCD}(\mathrm{PQCD})$ approach can be applied to the pion structure is expected to be lower than the case of the nucleon.

Recent discussions on the anti-de Sitter space geometry/conformal field theory(AdS/CFT) correspondence reveals a remarkable consistency with the QCD predictions on both hadron mass spectra and electromagnetic form factors [12, 13]. In particular, the power-law behavior of the pion form factor $F\left(Q^{2}\right) \sim 1 / Q^{2}$ is well reproduced by the AdS/CFT correspondence. The key ingredient in this correspondence is the conformal symmetry valid in the negligible quark masses. In this work, we confirm that the power-law behavior of the pion form factor is indeed attained by taking into account a momentum dependent dynamical quark mass which becomes negligibly small at large momentum region even in the nonperturbative quark model analysis. This result is consistent with an important point of the AdS/CFT prediction, namely the holographic wavefunction contains the contribution from all scales up to the confining scale. Thus, it is rather unnatural to distinguish the soft and hard pion form factors in comparing the predictions between QCD and AdS/CFT. However, due to the ongoing debate on the PQCD applicability in exclusive processes, it is of special interest in the QCD side to study the transition from the soft regime governed by all kinds of quark-gluon correlations at low $Q^{2}$ to the perturbative regime at high $Q^{2}$. We thus discuss a correlation between the shape of nonperturbative quark distribution amplitude(DA) and the amount of soft and hard contributions to the pion form factor utilizing our light-front quark model (LFQM) [14]. Similar to the previous findings from the Sudakov suppression [4, [5, 6,, 7$]$ of the soft contribution, we note that the soft and hard contributions are correlated with each other, i.e. the suppression of the endpoint region for the quark DA corresponds to the suppression of the soft contribution, or equivalently, the enhancement of the hard contribution. We further confirm that the higher helicity components suppress the contributions from the ordinary helicity components.

The paper is organized as follows. In the next section (Section II), we present the soft contribution to the pion form factor using the LFQM and discuss a consistency with the AdS/CFT correspondence. In section III, we discuss the hard contribution to the pion form factor including the intrinsic transverse momentum effect. In section IV, we show our numerical results of the nonperturbative quark model predictions on the pion DA and form factor as well as the PQCD prediction on the pion form factor. The correlation between soft and hard contributions and the higher helicity contribution are also discussed in this section. Summary and conclusion follow in Section V.

\section{PION FORM FACTOR IN LFQM}

To discuss nonperturbative quark model prediction of the pion form factor, we briefly summarize our LFQM 14] first. The model wave function for a pion [14] is given by

$$
\Psi\left(x, \mathbf{k}_{\perp}, \lambda \bar{\lambda}\right)=\phi_{R}\left(x, \mathbf{k}_{\perp}\right) \mathcal{R}_{\lambda \bar{\lambda}}\left(x, \mathbf{k}_{\perp}\right)
$$

where $\phi_{R}\left(x, \mathbf{k}_{\perp}\right)$ is the radial wave function and $\mathcal{R}_{\lambda \bar{\lambda}}\left(x, \mathbf{k}_{\perp}\right)$ is the spin-orbit wave function obtained by the interaction-independent Melosh transformation [15] from the ordinary equal-time static spin-orbit wave function assigned by the quantum numbers $J^{P C}=0^{-+}$. Here, $\lambda+\bar{\lambda}=0$ and 1 represent the contributions from the ordinary-helicity and the higher-helicity components, respectively. The detailed description for the spin-orbit wave function can be found in [14] as well as in other literatures [16]. The pion wave function $\Psi$ is represented by the Lorentz-invariant variables $x_{i}=p_{i}^{+} / P^{+}$, $\mathbf{k}_{\perp i}=\mathbf{p}_{\perp i}-x_{i} \mathbf{P}_{\perp}$ and $\lambda_{i}$, where $P, p_{i}$ and $\lambda_{i}$ are the meson momentum, the momenta and helicities of the constituent quarks, respectively. The radial wave function is 
given by

$$
\phi_{R}\left(x, \mathbf{k}_{\perp}\right)=\sqrt{\frac{1}{\pi^{3 / 2} \beta^{3}}} \exp \left(-\vec{k}^{2} / 2 \beta^{2}\right),
$$

where the gaussian parameter $\beta$ is related with the size of pion and the three momentum squared $\vec{k}^{2}$ in terms of the light-front $(\mathrm{LF})$ variables is given by

$$
\vec{k}^{2}=\frac{\mathbf{k}_{\perp}^{2}+m^{2}}{4 x(1-x)}-m^{2},
$$

for the quark mass $m_{u}=m_{d}=m$. Here, it is easy to see that the invariant mass of the pion $M_{0}=\sqrt{\frac{\mathbf{k}_{\perp}^{2}+m^{2}}{x(1-x)}}=$ $2 \sqrt{\vec{k}^{2}+m^{2}}$ and the longitudinal component $k_{z}$ of the three momentum is given by $k_{z}=(x-1 / 2) M_{0}$.

For the low momentum transfer phenomenology in LFQM, it is customary to take a constant constituent quark mass $m$ as a mean value of the momentum dependent dynamical quark mass at low momentum region. The momentum dependence of the dynamical quark mass in the spacelike momentum region has been discussed in lattice QCD [17] as well as in other approaches, especially in Dyson-Schwinger 18, 19 approach. Although the exact form of $m\left(\vec{k}^{2}\right)$ is still not known, the evolution of $m\left(\vec{k}^{2}\right)$ to a small current quark mass in large $\vec{k}^{2}$ region is generally agreed. Thus, for the large momentum transfer phenomenology in PQCD, it seems reasonable to take a negligible current quark mass, $m \approx 0$, for the pion form factor.

Matching between the low momentum LFQM prediction and the large momentum PQCD prediction is a highly nontrivial task which goes beyond the scope of our present work. Nevertheless, a sort of consistency between LFQM and PQCD predictions can be achieved by taking into account a momentum dependent dynamical quark mass in the following sense.

The PQCD factorization of the pion form factor $F_{\pi}\left(Q^{2}\right)$ can be found by taking a large $Q^{2}$ limit of the LFQM formula, i.e. the convolution of the initial and final LFQM wave functions. Since the LFQM wave function satisfies a LF bound-state equation, one can iterate the final wave function at the momentum scale $Q$ to yield an irreducible scattering kernel convoluted with a quark DA which is a transverse-momentum-integrated wave function collinear up to the scale $Q$. Similarly, the factorized initial quark DA is also at the scale $Q$ which is the probing momentum scale of the virtual photon. Thus, the factorized PQCD amplitude of $F_{\pi}\left(Q^{2}\right)$ is symmetric under the exchange of the initial and final quark DAs. This implies that the effective quark degrees of freedom probed by the virtual photon in computing the main contribution to $F_{\pi}\left(Q^{2}\right)$ has the momentum scale $Q$.

From this picture, one may parametrize the probed quark mass scale as the momentum scale $Q$ of the virtual photon and consider the effective dynamical quark mass as $m\left(Q^{2}\right)$. For the low and high $Q^{2}, m\left(Q^{2}\right)$ corresponds to the consituent and current quark mass, respectively. Thus, one may treat the quark mass in LFQM for the low $Q^{2}$ phenomenology as a consituent quark mass and the quark mass in PQCD for the high $Q^{2}$ phenomenology as a current quark mass. For simplicity in discussing the main features of soft and hard form factor, one may take each (consituent or current) mass as a constant mean value of $m\left(Q^{2}\right)$ in each (low or high) $Q$ region.

In our calculation of both soft and hard form factor, we adopt the Drell-Yan-West frame $\left(q^{+}=q^{0}+q^{3}=0\right)$ with $\mathbf{q}_{\perp}^{2}=Q^{2}=-q^{2}$. The momentum assignment in this $q^{+}=0$ frame for the pion form factor is given by $P=$ $\left(P^{+}, M_{\pi}^{2} / P^{+}, 0\right)$ and $q=\left(0, \mathbf{q}_{\perp}^{2} / P^{+}, \mathbf{q}_{\perp}\right)$. In this frame, the charge form factor of the pion, $\left\langle P+q\left|J^{\mu}\right| P\right\rangle=(2 P+$ $q)^{\mu} F_{\pi}\left(Q^{2}\right)$, can be expressed for the "+"-component of the current $J^{\mu}$ as follows [14]:

$$
\begin{aligned}
F_{\pi}^{\mathrm{LFQM}}= & \int_{0}^{1} d x \int d^{2} \mathbf{k}_{\perp} \sqrt{\frac{\partial k_{z}}{\partial x}} \phi_{R}\left(x, \mathbf{k}_{\perp}\right) \sqrt{\frac{\partial k_{z}^{\prime}}{\partial x}} \phi_{R}\left(x, \mathbf{k}_{\perp}^{\prime}\right) \\
& \times \frac{m^{2}+\mathbf{k}_{\perp} \cdot \mathbf{k}_{\perp}^{\prime}}{\sqrt{m^{2}+\mathbf{k}_{\perp}^{2}} \sqrt{m^{2}+\mathbf{k}_{\perp}^{\prime 2}}}
\end{aligned}
$$

where $\mathbf{k}_{\perp}^{\prime}=\mathbf{k}_{\perp}+(1-x) \mathbf{q}_{\perp}$ and the factors $m^{2}$ and $\mathbf{k}_{\perp} \cdot \mathbf{k}_{\perp}^{\prime}$ in the numerator come from the ordinary $\operatorname{helicity}(\lambda+\bar{\lambda}=0)$ and the higher helicity $(\lambda+\bar{\lambda}= \pm 1)$ components of the spin-orbit wave function $\mathcal{R}_{\lambda \bar{\lambda}}\left(x, \mathbf{k}_{\perp}\right)$, respectively. As discussed above, one should understand $m$ as a function of $Q^{2}$ in principle although in practice $m\left(Q^{2}\right)$ for the low $Q^{2}$ phenomenology can be taken as a constant constituent quark mass. For the constant constituent quark mass $m$, our LFQM prediction of the pion form factor provides a gaussian fall-off at high $Q^{2}$ region as expected from the gaussian form of our radial wave function $\phi_{R}\left(x, \mathbf{k}_{\perp}\right)$ given by Eq. (2). In this case, the AdS/CFT correspondence doesn't work due to the appreciable constituent quark mass which breaks the conformal symmetry. However, it is remarkable that $F_{\pi}\left(Q^{2}\right)$ in Eq. (4) reveals a power-law behavior even with our gaussian radial wave function $\phi_{R}\left(x, \mathbf{k}_{\perp}\right)$ if $m$ is replaced by $m\left(Q^{2}\right)$ yielding a negligible current quark mass at large $Q^{2}$. We note that $\phi_{R}\left(x, \mathbf{k}_{\perp}\right) \phi_{R}\left(x, \mathbf{k}_{\perp}^{\prime}\right)$ in Eq.(44) provides a mass-dependent weighting factor $e^{-\frac{m^{2}}{4 x(1-x) \beta^{2}}}$ which severely suppresses the contribution from the endpoint region of $x \rightarrow 0$ and 1 unless $m \rightarrow 0$. When the conformal symmetry limit $(m \rightarrow 0)$ is taken, however, there is no such suppression of the endpoint region and the high $Q^{2}$ behavior of the form factor dramatically changes from a gaussian fall-off to a power-law reduction. This may partly explain why the power-law behavior attained in our Eq.(4) is not accidental but a consequence of the constraint taken from the conformal symmetry. While our result $Q^{n} F_{\pi}\left(Q^{2}\right) \rightarrow$ const. confirms the recent findings of the AdS/CFT correspondence 12, 13], the power $n$, e.g. $n=2$ or 4 , still depends on the details of the LFQM calculation such as whether one takes into account the Jacobi factor or not in Eq.(44). The detailed numerical results are presented in section IV. 

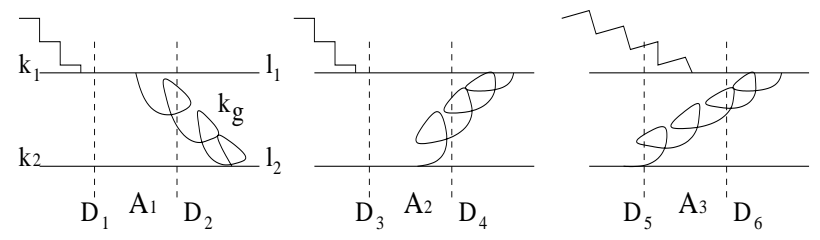

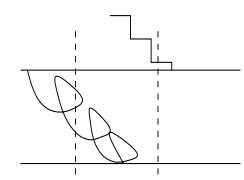

$\begin{array}{lllll}\mathrm{D}_{7} & \mathrm{~B}_{1} & \mathrm{D}_{8}\end{array}$

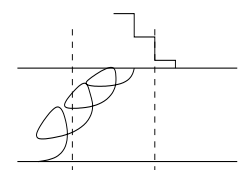

$\begin{array}{llll}\mathrm{D}_{9}^{\prime} & \mathrm{B}_{2} & \mathrm{D}_{10}\end{array}$

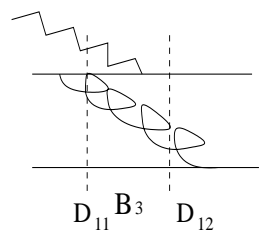

FIG. 1: Leading order light-front time-ordered diagrams for the pion form factor.

\section{HARD CONTRIBUTION IN PQCD}

At high momentum transfer, the pion form factor in leading order can be calculated within the PQCD from the soft part of the wave function by means of a homogeneous Bethe-Salpeter equation. Absorbing the perturbative kernel of the Bethe-Salpeter equation in a hard scattering amplitude $T_{H}$ containing all two-particle irreducible amplitudes for $\gamma^{*}+q \bar{q} \rightarrow q \bar{q}$, the hard contribution to the pion electromagnetic form factor is given by

$$
F_{\pi}^{\mathrm{PQCD}}\left(Q^{2}\right)=\int \frac{d^{3} k d^{3} l}{16 \pi^{3}} \phi_{R}\left(y, \mathbf{l}_{\perp}\right) \mathcal{T}_{H} \phi_{R}\left(x, \mathbf{k}_{\perp}\right),
$$

where $d^{3} k=d x d^{2} \mathbf{k}_{\perp} \sqrt{\partial k_{z} / \partial x}\left(d^{3} l=d y d^{2} \mathbf{l}_{\perp} \sqrt{\partial l_{z} / \partial y}\right)$ and $\mathcal{T}_{H}$ is related to the original $T_{H}$ including the spinorbit wave function, i.e.

$$
\mathcal{T}_{H}=\mathcal{R}_{0} T_{H}^{(\lambda+\bar{\lambda}=0)}+\mathcal{R}_{ \pm 1} T_{H}^{(\lambda+\bar{\lambda}= \pm 1)},
$$

with $\mathcal{R}_{0( \pm 1)}=\mathcal{R}_{\uparrow \downarrow(\uparrow \uparrow)}^{*}\left(y, \mathbf{l}_{\perp}\right) \mathcal{R}_{\uparrow \downarrow(\uparrow \uparrow)}\left(x, \mathbf{k}_{\perp}\right)+(\uparrow \leftrightarrow \downarrow)$. The hard scattering amplitudes $T_{H}^{(\lambda+\bar{\lambda}=0)}$ and $T_{H}^{(\lambda+\bar{\lambda}= \pm 1)}$ in Eq. (6) represent the contributions from the ordinaryhelicity and the higher-helicity components, respectively.

To lowest order in perturbation theory, the hard scattering amplitude $T_{H}$ is to be calculated from the timeordered one-gluon-exchange diagrams shown in Fig. 1, The internal momenta for $(+, \perp)$-components are given by $k_{1}=\left(x_{1} P_{1}^{+}, \mathbf{k}_{\perp}\right), \quad k_{2}=\left(x_{2} P_{1}^{+},-\mathbf{k}_{\perp}\right), \quad l_{1}=$ $\left(y_{1} P_{1}^{+}, y_{1} \mathbf{q}_{\perp}+\mathbf{l}_{\perp}\right)$, and $l_{2}=\left(y_{2} P_{1}^{+}, y_{2} \mathbf{q}_{\perp}-\mathbf{l}_{\perp}\right)$, where $x_{1}=x, x_{2}=1-x, y_{1}=y$, and $y_{2}=1-y$.

The explicit forms for the hard scattering amplitudes including the higher twist effects such as the quark mass $m$ and the intrinsic transverse momenta $\mathbf{k}_{\perp}$ and $\mathbf{l}_{\perp}$ have been presented in Ref. [20]. There seems no need to rewrite them in this work since the only difference in this work is to take the quark mass as a negligibly small current quark mass, $m \approx 0$. Including the intrinsic transverse momenta, the leading contribution of the hard scattering amplitude for the higher helicity components is of
$1 / Q^{4}$, which is the next-to-leading contribution. When the intrinsic transverse momenta are neglected, we find by the power counting that the higher helicity contributions goes to zero and the hard scattering amplitude for ordinary helicity components reduces to the usual leading twist result:

$$
T_{H}=\frac{16 \pi \alpha_{s} C_{F}}{Q^{2}}\left(\frac{e_{u}}{x_{2} y_{2}}+\frac{e_{\bar{d}}}{x_{1} y_{1}}\right),
$$

where $\alpha_{s}=4 \pi /\left[\left(11-2 n_{f} / 3\right) \log \left(Q^{2} / \Lambda^{2}\right)\right]$ is the QCD running coupling constant and $C_{F}(=4 / 3)$ is the color factor. For the pion form factor in PQCD for large $Q^{2}$, we neglect the terms not only the quark mass $m$ but also $\mathbf{k}_{\perp}^{2} / \mathbf{q}_{\perp}^{2}, \mathbf{l}_{\perp}^{2} / \mathbf{q}_{\perp}^{2}$, and $\mathbf{k}_{\perp} \cdot \mathbf{l}_{\perp} / \mathbf{q}_{\perp}^{2}$ both in the energy denominators and the numerators of the hard scattering amplitude $T_{H}$ due to $\mathbf{k}_{\perp}^{2} \ll \mathbf{q}_{\perp}^{2}$ and $\mathbf{l}_{\perp}^{2} \ll \mathbf{q}_{\perp}^{2}$. Our analytic forms for the hard scattering amplitudes are the same as those obtained by Huang et.al [9]. However, in choosing the radial wave function as a nonperturbative input, we include the Jacobi factor $\sqrt{M_{0} / 4 x(1-x)}$ while the authors in [9] do not. This makes quantitative difference for the soft and hard form factors between the ones in [9] and ours. Although the qualitative behaviors are equivalent to each other between the two(ours and Ref. [9]) at high $Q^{2}$ in PQCD, we discuss the effect of the Jacobi factor with respect to the AdS/CFT correspondence in our numerical results.

\section{NUMERICAL RESULTS}

The key idea in our LFQM [14] is to treat the gaussian radial wave function as a trial function for the variational principle to the QCD-motivated Hamiltonian saturating the Fock state expansion by the constituent quark and antiquark, i.e. $H_{q \bar{q}}=H_{0}+V_{\text {int }}$, where the interaction potential $V_{\text {int }}$ consists of confining and hyperfine interaction terms. For the numerical calculations, we obtained two different sets of model parameters, i.e. $(1)(m, \beta)=$ $(0.25,0.3194) \mathrm{GeV}$ for the harmonic oscillator(HO) confining potential, and $(2)(m, \beta)=(0.22,0.3659) \mathrm{GeV}$ for the linear confining potential, from our variational principle for the QCD-motivated effective Hamiltonian [14]. Both parameter sets have shown to provide a good agreement with the available experimental data for form factors, decay constants and charge radii etc. of various pseudoscalar and vector mesons as well as their radiative decay widths [14].

The quark DA of pion, $\phi(x, \mu)$, i.e. the probability of finding collinear quarks up to the scale $\mu$ in the $L_{z}=0(s-$ wave) projection of the pion wave function [1] is defined by

$$
\phi(x, \mu)=\int^{\mathbf{k}_{\perp}^{2}<\mu^{2}} \frac{d^{2} \mathbf{k}_{\perp}}{\sqrt{16 \pi^{3}}} \sqrt{\frac{\partial k_{z}}{\partial x}} \Psi\left(x, \mathbf{k}_{\perp}, \lambda \bar{\lambda}\right),
$$

where $\Psi\left(x, \mathbf{k}_{\perp}, \lambda \bar{\lambda}\right)=\phi_{R}\left(x, \mathbf{k}_{\perp}\right)\left(\mathcal{R}_{\uparrow \downarrow}-\mathcal{R}_{\downarrow \uparrow}\right) / \sqrt{2}$ and the higher helicity components $(\uparrow \uparrow$ and $\downarrow \downarrow)$ do not contribute 


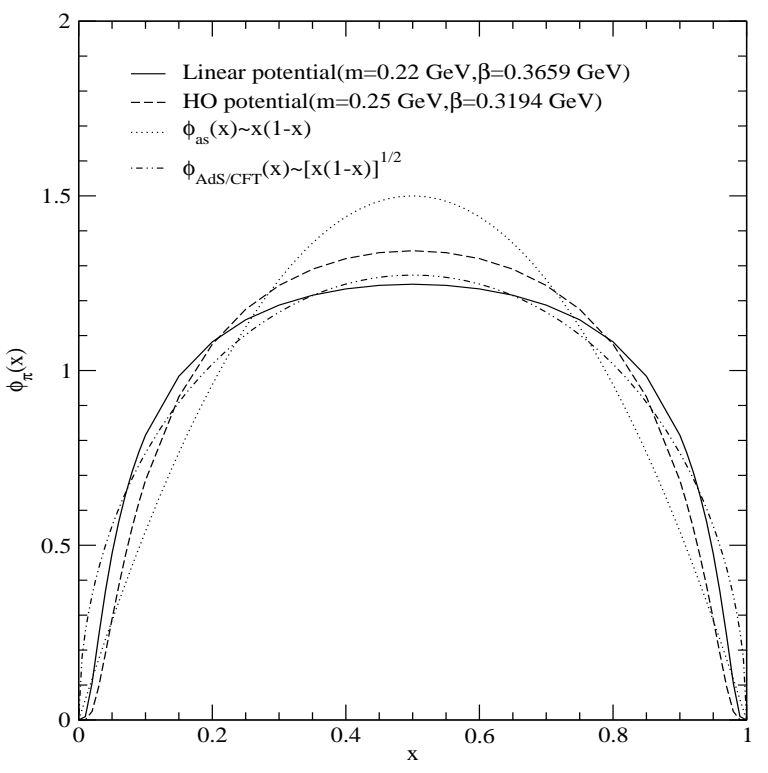

FIG. 2: The distribution amplitude for the pion using two different sets of model parameters, $(1)(m, \beta)=(0.25,0.3194)$ $\mathrm{GeV}$ (dotted line), and $(2)(m, \beta)=(0.22,0.3659) \mathrm{GeV}$ (solid line) compared with the usual QCD asymptotic DA(dotted line) as well as the AdS/CFT prediction of the asymptotic DA(double-dot-dashed line).

to DA in this case. Here, again the quark mass in Eq. (8) is the renormalization scale $\mu$ dependent. However, for the LFQM phenomenology at low momentum scale, one may effectively take $m(\mu)$ as a constant constituent quark mass.

In Fig. 2, we show the normalized quark DAs of the pion obtained from the linear(solid line) and $\mathrm{HO}$ (dashed line) potential parameters, respectively, in comparison with the usual QCD asymptotic DA, $\phi_{a s}(x)=6 x(1-$ $x)$ (dotted line), and the AdS/CFT prediction of the asymptotic DA, $\phi_{\operatorname{AdS} / C F T}(x)=\frac{8}{\pi} \sqrt{x(1-x)}$ (doubledot-dashed line). While the quark DA for the HO potential parameter set lies between $\phi_{a s}$ and $\phi_{A d S / C F T}$, that for the linear confining potential gets very close to $\phi_{A d S / C F T}$. In other words, the central(end) point region is rather enhanced(suppressed) for the HO potential parameter set (1) than the linear potential paramter set (2). From our model calculation, we find that the shape of the quark DA becomes sharper and more suppressed at the endpoint region as the constituent quark mass(gaussian parameter $\beta$ ) increases(decreases). As a sensitivity check, we note that the central point of the quark DA for the set (1) varies about $3.7 \%(2.2 \%)$ by changing $10 \%$ of the quark mass ( $\beta$ value). This indicates that our results for the quark DA are quite stable for the variation of model parameters.

In Fig. 3, we show our previously published result [14] for $Q^{2} F_{\pi}\left(Q^{2}\right)$ obtained from our linear(solid line) and $\mathrm{HO}$ (dashed line) confining potential models and compare

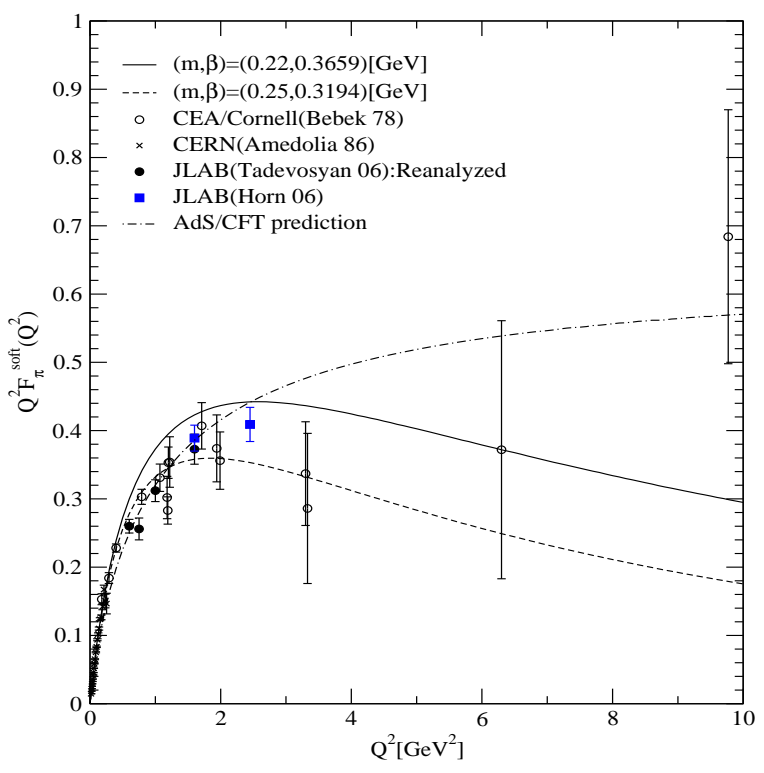

FIG. 3: Soft pion form factor $Q^{2} F_{\pi}\left(Q^{2}\right)$ in our linear(solid line) and $\mathrm{HO}$ (dashed line) potential models compared with AdS/CFT prediction(dot-dashed line) as well as data 22, 23, 24, 25]. Tadevosyan et al. 25] have reanalyzed previously published data by Volmer et al. [26] and obtained the data points represented by the filled circles.

both with the AdS/CFT prediction(dot-dashed line) 21 and the data 22, 23, 24, 25], which includes the most recent results from JLAB [24, 25]. As expected, the gaussian fall-off at high $Q^{2}$ region provided by our previous LFQM results 14] with $m=0.22 \mathrm{GeV}$ (solid line) and $m=0.25 \mathrm{GeV}$ (dashed line) cannot match with the power-law behavior of the AdS/CFT prediction (dotdashed line) although all the lines (solid, dashed, dotdashed) are comparable for $Q^{2}$ up to a few $\mathrm{GeV}^{2}$. While the recent JLAB data from Horn et al. 24 extracted two new values at $Q^{2}=1.60$ and $2.45 \mathrm{GeV}^{2}$, Tadevosyan et al. 25] have reanalyzed the previously published values [26] of $Q^{2}=0.6-1.6 \mathrm{GeV}^{2}$ and obtained rather lower values than those in Ref. [26]. We should note that our model parameters have been determined without the use of the pion form factor $F_{\pi}$ data but from the variational principle for the QCD-motivated effective Hamiltonian to fit the meson mass spectra. While our HO potential model(dashed line) provides a very good description of the reanalyzed values from JLAB 25] up to $Q^{2}=1.60 \mathrm{GeV}^{2}$, two newly extracted values from 24](filled squares) seem closer to our result from the linear potential model(solid line) or the AdS/CFT prediction(dot-dashed line). As shown in 24, 25], various model predictions such as Dyson-Schwinger [19], QCD Sum Rules [27], and dispersion relation [28] including our linear potential model slightly overestimate the pion form factor compare to the new JLAB data [24, 25]. Although it may be difficult to pin down which of our models (linear 


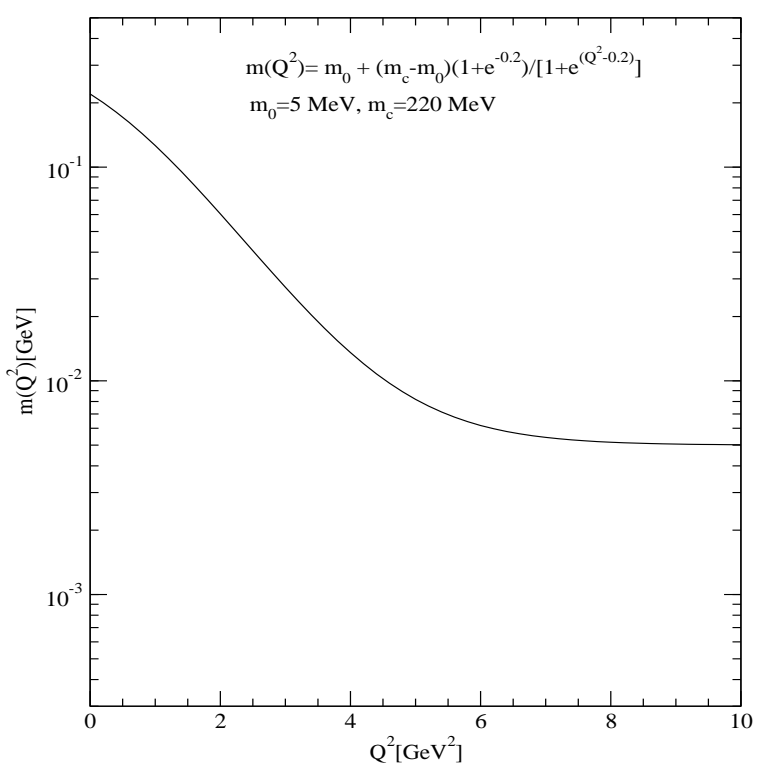

FIG. 4: Quark mass evolution $m\left(Q^{2}\right)$ in spacelike momentum region. The $m_{0}$ and $m_{c}$ represent the current(at high $Q^{2}$ ) and constituent(at low $Q^{2}$ ) quark masses, respectively.

or $\mathrm{HO}$ ) is better to describe the current available data, we at least note that the suppression of the quark DA at the end points region corresponds to the suppression of the soft contribution to the pion form factor at low $Q^{2}$ region. This is rather similar to the case of the Sudakov suppression enhancing the hard contribution to the form factor [4, 5, 6, 6].

To investigate a consistency with the recent findings from the AdS/CFT correspondence [12, 21], we consider a momentum-dependent quark mass for the calculation of $F_{\pi}\left(Q^{2}\right)$ in LFQM. Since it is beyond the scope of our work to find an explicit form of $m\left(Q^{2}\right)$, we take a simple parametrization similar to what we have already used in our previous work [29] as shown in Fig. [4 The details of discussion for this type of parametrization and the consistency with the PCAC relation [30] can be found in Ref. 29]. For comparison, we also simply take a negligible current quark mass $m=0$ respecting the conformal symmetry in Eq. (44) and show both results in Fig. 5.

In Fig. 5, the solid line is the result of Eq.(4) using the effective dynamical quark mass $m\left(Q^{2}\right)$ drawn in Fig 4 which respects the conformal symmetry at high $Q^{2}$ limit. In comparison, the dashed line is the result of Eq.(4) taking $m=0$ and the dot-dashed line is the AdS/CFT prediction presented in Ref. 21]. The corresponding values of the gaussian parameter $\beta=0.17 \sim 0.18 \mathrm{GeV}$ are not much different from the gaussian parameter $\kappa=$ $0.4 \mathrm{GeV}$ used in a holographic AdS Gaussian-modifiedmetric model[21], e.g., the correspondence between $\beta$ and $\kappa$ can be easily seen from the gaussian dependence of our model wave function $\Psi\left(x, \mathbf{k}_{\perp}\right) \sim e^{-\mathbf{k}_{\perp}^{2} / 2(2 \beta)^{2} x(1-x)}$ in $m=0$ limit and the AdS Gaussian-modified-metric

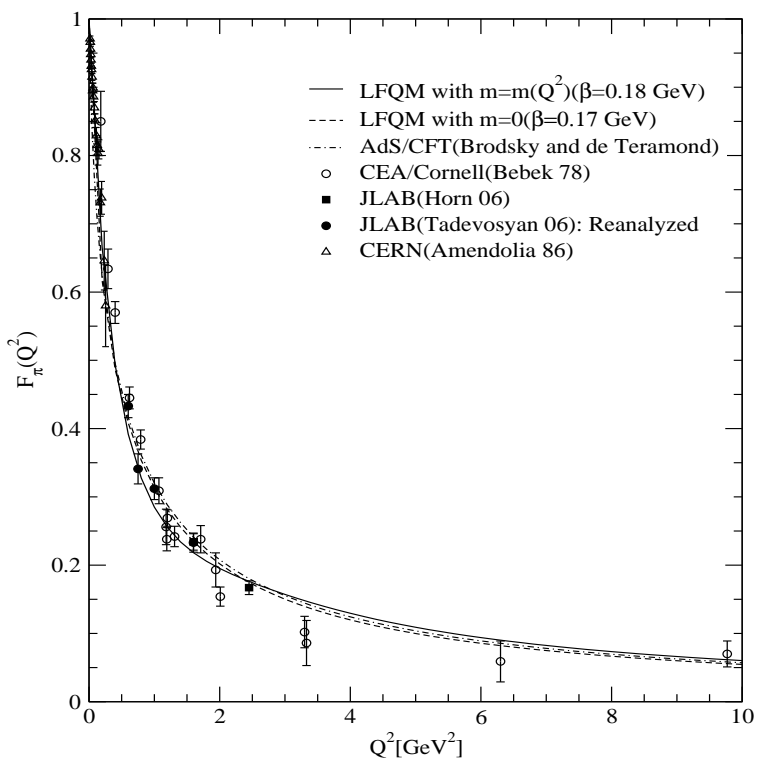

FIG. 5: $\quad F_{\pi}\left(Q^{2}\right)$ with running mass $m\left(Q^{2}\right)$ (solid line) and negilgible mass $m=0$ (dashed line) in our LFQM (Eq.(4)) compared to the AdS/CFT result [21](dot-dashed line).

model wave function $\Psi\left(x, \mathbf{k}_{\perp}\right) \sim e^{-\mathbf{k}_{\perp}^{2} / 2 \kappa^{2} x(1-x)}$ 31]. The decay constants obtained with these gaussian parameters are also not much different from the experimental value $f_{\pi}=92.4 \mathrm{MeV}$. As shown in Fig 5 , our results respecting the conformal symmetry are in excellent agreement with the AdS/CFT prediction 21]. This implies that, in the massless quark case, our gaussian wave function with the Jacobi factor leads to the scaling behavior $F_{\pi}\left(Q^{2}\right) \sim 1 / Q^{2}$ as $Q^{2} \rightarrow \infty$, being consistent with the scaling behavior obtained from AdS/CFT correspondence [12] at large $Q^{2}$. Although the power-law behavior $Q^{n} F_{\pi}\left(Q^{2}\right) \rightarrow$ const. for large $Q^{2}$ is still obtained even without the Jacobi factor, we find $n=4$ rather than $n=2$ if the Jacobi factor is not taken into account. The asymptotic behavior of $n=4$ for the meson form factor with spin- $1 / 2$ quarks has recently been found using meromorphization for spin- $1 / 2$ quarks 13 . Our work here indicates that the power $n$, however, does depend on whether one takes into account the Jacobi factor or not in the LFQM calculation. Our result shows that $n=2$ can be attained by taking into account the Jacobi factor in Eq. (4). This also distinguishes our analysis from Ref. 9]. Other effects of the presence/absence of the Jacobi factor to various static properties of mesons can be found in Ref. [32].

In Fig. 6] we show the hard(PQCD) and soft(LFQM) contributions to the pion form factor $Q^{2} F_{\pi}\left(Q^{2}\right)$ obtained from the $\mathrm{HO}[\mathrm{top}]$ and linear[bottom] potential parameters, respectively. The solid and dotted lines represent the soft and hard(ordinary+higher helicities) contributions to the pion form factor, respectively. We include each helicity component contributions, i.e. the 

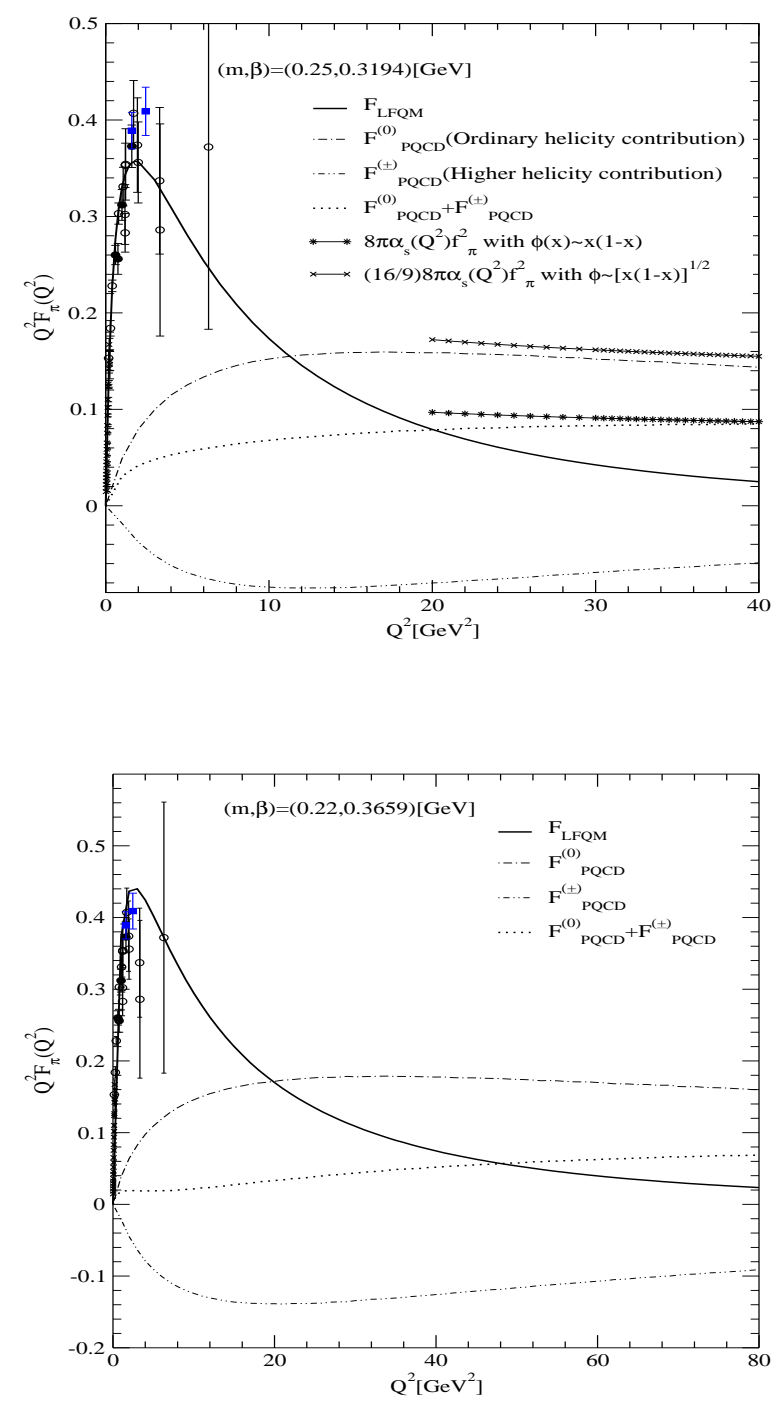

FIG. 6: The hard(PQCD) and soft(LFQM) contributions to the pion form factor $Q^{2} F_{\pi}\left(Q^{2}\right)$ using two different sets of model parameters, $(1)(m, \beta)=(0.25,0.3194) \mathrm{GeV}$ [top], and (2) $(m, \beta)=(0.22,0.3659) \mathrm{GeV}[$ bottom].

ordinary(dot-dashed line) and higher(doubledot-dashed line) helicity contributions. In the top panel, we also show the leading twist PQCD predictions using both $\phi_{a s}$ and $\phi_{A d S / C F T}$ for the comparison. As noted in Ref.[21], the broader shape of $\phi_{A d S / C F T}$ increases the magnitude of the leading twist PQCD prediction for the pion form factor by a factor of $16 / 9$ compared to the prediction based on the asymptotic form. The new experimental data are taken from [24, 25]. In the AdS/CFT side, it is still an open question how the AdS/CFT prediction of $Q^{2} F_{\pi}\left(Q^{2}\right)$ shown in Fig 3 can approach asymptotically to the PQCD prediction using $\phi_{\operatorname{AdS} / C F T}$ shown in Fig[6 [31]. Possibly, the higher order PQCD corrections may reconcile the two approaches and in addition one may need to evolve the AdS/CFT DA to the scale of the gluon virtuality. Since the loop contributions are not included to the the present approximation of the AdS/CFT correspondence, quantum corrections are to be incorporated further [31]. It is not yet clear how to do this in practice, although there is much interest in the theme [33]. In the QCD side, however, there are a few things to note from Fig. 6 regarding on the soft/hard contribution: (a) The soft contribution for the linear potential parameters is larger than that for the HO potential ones. (b) The hard contribution for the linear potential parameters is smaller than that for the $\mathrm{HO}$ potential ones. (c) The higher helicity components suppress the contributions from the ordinary helicity components. From (a) and (b), we find that the soft and hard contributions are correlated to each other, i.e. as the endpoint region for the quark DA is more suppressed, the soft(hard) contribution to the pion form factor does get less(more) enhancement. This finding is rather similar to the previous findings from the Sudakov suppression of the soft contribution [4, 5, 6, 7]. From (c), we also see that the intrinsic transverse momentum effect included in this work is still effective even at $Q^{2} \approx 80 \mathrm{GeV}^{2}$ range.

\section{SUMMARY AND CONCLUSION}

Due to the ongoing debate on the PQCD applicability in exclusive processes, we studied the soft and hard contributions to the pion form factor utilizing both LFQM and PQCD approaches.

We discussed a constraint of conformal symmetry in the analysis of the pion form factor. The usual powerlaw behavior of the pion form factor obtained in the perturbative QCD analysis can also be attained by taking negligible quark masses in the nonperturbative quark model analysis, confirming the recent AdS/CFT correspondence. Inclusion of the Jacobi factor with the gaussian radial wave function in the LFQM analysis is essential to attain the power-law behavior $F_{\pi}\left(Q^{2}\right) \sim 1 / Q^{2}$ in the massless quark limit. We find that the correlation between the shape of nonperturbative quark distribution amplitude and the amount of soft and hard contributions to the form factor, i.e. the suppresion(enhancement) of the endpoint region for the quark distribution amplitude corresponds to the suppression of the soft(hard) contribution. The fact that the higher helicity components suppress the contributions from the ordinary helicity components are also confirmed in this work.

The conformal symmetry as well as the correlation between soft and hard contributions may provide a useful constraint on the model building for the form factor analysis.

\section{Acknowledgments}

We thank Stanley J. Brodsky and Guy F. de Teramond for many useful discussions. This work was supported by 
a grant from the U.S. Department of Energy(No. DEFG02-96ER40947). H.-M. Choi was supported in part by Korea Research Foundation under the contract KRF2005-070-C00039. The National Energy Research Scientific Center is also acknowledged for the grant of super- computing time. We also acknowledge the Asia Pacific Center for Theoretical Physics(APCTP) for the support on the APCTP Focus Program during which this work was completed.
[1] G. P. Lepage and S. J. Brodsky, Phys. Rev. D 22, 2157 (1980).

[2] N. Isgur and C. H. Llewellyn Smith, Phys. Rev. Lett. 52, 1080 (1984); Nucl. Phys. B 317, 526 (1989).

[3] A. V. Radyushkin, Nucl. Phys. A 532, 141 (1991).

[4] H. N. Li and G. Sterman, Nucl. Phys. B 381, 129 (1992); H. N. Li, Phys. Rev. D 48, 4243 (1993).

[5] O.C. Jacob and L.S. Kisslinger,Phys. Rev. Lett. 56, 225 (1986); Phys. Lett. B 243, 323 (1990).

[6] R. Jakob and P. Kroll, Phys. Lett. B 315, 463 (1993).

[7] V. Anikin, A.-E. Dorokhov and L. Tomio, Phys. Lett. B 475, 361 (2000).

[8] N.G. Stefanis, W. Schroers, and H.-C. Kim, Eur. Phys. J.C 18, 137 (2000).

[9] T. Huang, X.-G. Wu, and X.-H. Wu, Phys. Rev. D 70, 053007 (2004).

[10] C.-R. Ji, A. Pang, and A. Szcepaniak, Phys. Rev. D 52, 4038 (1995).

[11] A. Szcepaniak, C.-R. Ji, and A. Radyushkin, Phys. Rev. D 57, 2813 (1998).

[12] S. J. Brodsky and Guy F. de Teramond, Phys. Lett. B 582, 211 (2004); Phys. Rev. Lett. 96, 201601 (2006).

[13] A. V. Radyushkin, hep-ph/0605116

[14] H.-M. Choi and C.-R. Ji, Phys. Rev. D 59, 074015 (1999).

[15] H. J. Melosh, Phys. Rev. D 9, 1095 (1974).

[16] P. J. O'Donnell, Q.P. Xu, and H.K.K. Tung, Phys. Rev. D 52, 3966 (1995); W. Jaus, Phys. Rev. D 53, 1349 (1996); I.L. Grach, I.M. Narodetskii, and S. Simula, Phys. Lett. B 385, 317 (1996); H.-Y. Cheng, C.-Y.
Cheng, and C.-W. Hwang, Phys. Rev. D 55, 1559 (1997).

[17] J.I. Skullerud and A.G. Williams, Phys. Rev. D 63, 054508 (2001); J.B. Zhang et al., Phys. Rev. D 70, 034505 (2004).

[18] P. Maris and C.D. Roberts, Phys. Rev. C 58, 3659 (1998).

[19] P. Maris and P.C. Tandy, Phys. Rev. C 62, 204 (2000).

[20] H.-M. Choi and C.-R. Ji, Phys. Rev. D 73, 114020 (2006).

[21] G. F. de Teramond, Talk entitled "Mapping AdS/CFT Results for Holographic QCD to the Light Front" presented in the Workshop LC2006 (Light-Cone QCD and Nonperturbative Hadron Physics), Minneapolis, May 15-19, 2006; S. J. Brodsky, hep-ph/0608005 and hep-ph/0610115

[22] C. J. Bebek, et al., Phys. Rev. D 17, 1693 (1978).

[23] S.R. Amendolia, et al., Nucl. Phys. B 277, 168 (1986).

[24] T. Horn, et al., nucl-ex/0607007.

[25] V. Tadevosyan, et al., nucl-ex/0607007

[26] J. Volmer et al., Phys. Rev. Lett. 86, 1713 (2001).

[27] V. A. Nesterenko and A.V. Radyushkin, Phys. Lett. B 115, 410 (1982).

[28] B.V. Geshkenbein, Phys. Rev. D 61, 033009 (2000).

[29] L.S. Kisslinger, H.-M. Choi and C.-R. Ji,

[30] M.Gell-Mann, R.J.Oakes and B.Renner, Phys. rev. 175, 2195 (1968). Phys. Rev. D 63, 113005 (2001).

[31] Private communications with S. J. Brodsky and G. F. de Teramond.

[32] H.-M. Choi and C.-R. Ji, Phys. Rev. D 56, 6010 (1997).

[33] C. Csaki and M. Reece, hep-ph/0608266 